\title{
A natural localization of Hardy spaces in several complex variables
}

\author{
by Mihai Putinar (Riverside, Calif.) \\ and Roland WolfF (Münster and Saarbrücken) \\ Dedicated to the memory \\ of Professor Wtodzimierz Mlak
}

\begin{abstract}
Let $H^{2}(b \Omega)$ be the Hardy space of a bounded weakly pseudoconvex domain in $\mathbb{C}^{n}$. The natural resolution of this space, provided by the tangential Cauchy-Riemann complex, is used to show that $H^{2}(b \Omega)$ has the important localization property known as Bishop's property $(\beta)$. The paper is accompanied by some applications, previously known only for Bergman spaces.
\end{abstract}

1. Introduction. A fundamental question in abstract spectral theory is whether, given an operator or a system of operators, the underlying Hilbert space can be localized with respect to parts of the spectrum. Any spectral decomposition construction actually answers this question in specific terms. A branch of modern operator theory, called local spectral theory, studies different aspects of this localization problem.

Quite recently, a general framework based on sheaf theory and homological algebra techniques was proposed as a simplifying and unifying language in local spectral theory. Several classical contributions to the field (due to N. Dunford and J. T. Schwartz [6], E. Bishop [1], C. Foiaş [8]) as well as recent researches (see for instance the monograph of Vasilescu [17]) have been naturally included in this framework. The monograph [7] is devoted

1991 Mathematics Subject Classification: 47A13, 46M20, 32A35.

Key words and phrases: weakly pseudoconvex domain, Hardy space, quasi-coherent module, Bishop's property $(\beta)$, Toeplitz operators.

The research of the first author was supported by the National Science Foundation grant DMS9500954.

The research of the second author was supported by a grant from the German Academic Exchange Service. The material contained in this article is intended to be part of this author's Ph.D. thesis at the Westfälische Wilhelms-Universität at Münster, Germany. 
to this new approach to local spectral theory. (Most of the references in our paper will be forwarded to [7] rather than the original papers, all quoted and commented in [7].)

Let $H$ be a Hilbert $\mathcal{O}\left(\mathbb{C}^{n}\right)$-module, or, equivalently, a system of $n$ commuting linear bounded operators acting on $H(n \geq 1)$. It turns out that, in terms of topological modules, the spectral localization property of $H$ is equivalent to the existence of an analytic sheaf $\mathcal{F}$ of Fréchet spaces on $\mathbb{C}^{n}$ with $\mathcal{F}\left(\mathbb{C}^{n}\right) \cong H$ (as topological $\mathcal{O}\left(\mathbb{C}^{n}\right)$-modules) and with a good cohomological behaviour known as quasi-coherence in analytic geometry or, equivalently, as Bishop's property $(\beta)$ in operator theory. Various characterizations of this class of analytic sheaves are known, for instance the topological coherence (i.e. the existence of topologically free resolutions to the left) or the existence of an abstract Dolbeault resolution (i.e. a finite resolution to the right with soft analytic Fréchet sheaves).

Once a Hilbert $\mathcal{O}\left(\mathbb{C}^{n}\right)$-module $H$ is localizable in the above sense, the spectral theory of multiplication operators on $H$ with analytic functions is much simplified. Questions such as the computation of Taylor's joint (or essential joint) spectrum, the evaluation of the Fredholm index or the division of vector-valued distributions by operator-valued analytic functions were successfully studied with the homological-topological methods which are specific to localizable modules. See [7] for details with references.

So far, the typical example of a localizable analytic module was the Bergman space of a bounded pseudoconvex domain in $\mathbb{C}^{n}$; see [7], Chapter 8. The aim of the present paper is to prove that the Hardy space of a bounded weakly pseudoconvex domain in $\mathbb{C}^{n}$ is localizable. The proof of this fact relies, besides standard homological techniques, on the estimates for the tangential $\bar{\partial}$-operator obtained in the last two decades by G. Henkin [9], J. J. Kohn [10] and very recently improved by M.-C. Shaw [15]. Thus the main result of our article asserts that there is a (unique) quasi-coherent Fréchet $\mathcal{O}\left(\mathbb{C}^{n}\right)$-module which localizes the Hardy space of a weakly pseudoconvex domain. Actually, a precise description of this sheaf is available; see Section 3. Without developing all possible consequences of this result for the spectral analysis of Toeplitz operators with bounded analytic symbols, we put together in a separate section some applications which are easily obtained from the comparison with the case of Bergman spaces.

The paper is organized as follows. After this introduction, we recall in the second section some definitions and statements that are needed in the following sections. The third section is devoted to the main result, and the applications are given in the fourth and last section. 
2. Preliminaries. In this section we collect some facts, needed in the following, from topological homology theory and local spectral theory. For a complete introduction to this subject we refer to [7], where all the listed facts are proved. Further, we give a brief summary of the definition and properties of the tangential $\bar{\partial}$-operator and of Hardy spaces in several complex variables, as far as they are used in this article.

2.1. The topological torsion functor. For two locally convex spaces $E$ and $F$ we denote by $E \widehat{\otimes} F$ their completed $\pi$-tensor product (over $\mathbb{C}$ ). Let $\mathcal{A}$ be a Fréchet algebra with unit and assume that $E$ and $F$ are Fréchet $\mathcal{A}$-modules. Similarly to algebraic homology, we define $\widehat{\text { Tor }}$ to be the derived functor of $\widehat{\otimes}_{\mathcal{A}}$; more precisely, we denote by $\mathbf{B}_{\bullet}^{\mathcal{A}}(E, F)$ the Bar complex associated with $E$ and $F$ and define

$$
\begin{aligned}
\widehat{\operatorname{Tor}}_{0}^{\mathcal{A}}(E, F)=E \widehat{\otimes}_{\mathcal{A}} F & :=H_{0}\left(\mathbf{B}_{\bullet}^{\mathcal{A}}(E, F)\right), \\
\widehat{\operatorname{Tor}}_{p}^{\mathcal{A}}(E, F) & :=H_{p}\left(\mathbf{B}_{\bullet}^{\mathcal{A}}(E, F)\right) \quad \text { for } p \geq 1 .
\end{aligned}
$$

Here $H_{p}$ denotes the $p$ th homology space. The Tor-spaces are equipped with the quotient locally convex topology, which may not be Hausdorff. As in the algebraic case, any ( $\mathbb{C}$-split, topologically-) free resolution can be used to compute these spaces.

The characteristic property of the Tor-functor is contained in the following theorem.

TheOREM 2.1. Let $\mathcal{A}$ be a commutative Fréchet algebra with unit, $F$ a Fréchet $\mathcal{A}$-module and let

$$
0 \rightarrow E_{1} \rightarrow E_{2} \rightarrow E_{3} \rightarrow 0
$$

be an exact sequence of Fréchet $\mathcal{A}$-modules. If $\mathcal{A}$ and $F$ are nuclear, then the induced long sequence of locally convex spaces

$$
\begin{aligned}
\quad \ldots \rightarrow \widehat{\operatorname{Tor}}_{1}^{\mathcal{A}}\left(E_{1}, F\right) \rightarrow \widehat{\operatorname{Tor}}_{1}^{\mathcal{A}}\left(E_{2}, F\right) \rightarrow \widehat{\operatorname{Tor}}_{1}^{\mathcal{A}}\left(E_{3}, F\right) \\
\\
\rightarrow E_{1} \widehat{\otimes}_{\mathcal{A}} F \rightarrow E_{2} \widehat{\otimes}_{\mathcal{A}} F \rightarrow E_{3} \widehat{\otimes}_{\mathcal{A}} F \rightarrow 0
\end{aligned}
$$

is exact.

The nuclearity of $\mathcal{A}$ and $F$ is not necessary for the validity of Theorem 2.1 , since there are other conditions that guarantee it.

For later use we give the following definition.

Definition 2.2. Let $\mathcal{A}$ be a Fréchet algebra with unit. Two Fréchet $\mathcal{A}$-modules $E$ and $F$ are called transversal (in symbols $E \perp_{\mathcal{A}} F$ ) if

$$
E \widehat{\otimes}_{\mathcal{A}} F \text { is a Hausdorff locally convex space }
$$

and if

$$
\widehat{\operatorname{Tor}}_{p}^{\mathcal{A}}(E, F)=0 \quad \text { for } p \geq 1
$$


We conclude this subsection with some remarks to our main application. We deal primarily with the case $\mathcal{A}=\mathcal{O}\left(\mathbb{C}^{n}\right)$ and $E=\mathcal{O}(U)$ or $E=\mathcal{E}(U)$ with $U \subseteq \mathbb{C}^{n}$ open. All these spaces are nuclear Fréchet spaces, and we can use the Koszul resolution

$$
\mathbf{K}_{\bullet}(z-w, E \widehat{\otimes} F) \rightarrow F \rightarrow 0
$$

to compute the spaces $\widehat{\operatorname{Tor}}_{p}^{\mathcal{O}\left(\mathbb{C}^{n}\right)}(E, F)$ for a Fréchet $\mathcal{O}\left(\mathbb{C}^{n}\right)$-module $F$. In particular, we see in these cases that

$$
\widehat{\operatorname{Tor}}_{n}^{\mathcal{O}\left(\mathbb{C}^{n}\right)}(E, F) \text { is separated }
$$

and

$$
\widehat{\operatorname{Tor}}_{p}^{\mathcal{O}\left(\mathbb{C}^{n}\right)}(E, F)=0 \quad \text { for } p>n .
$$

2.2. Quasi-coherence and property $(\beta)$. Although the theory studied in this section makes sense in a more general context, we restrict ourselves to the special analytic space $\mathbb{C}^{n}$ with the corresponding sheaf $\mathcal{O}_{\mathbb{C}^{n}}$ of analytic

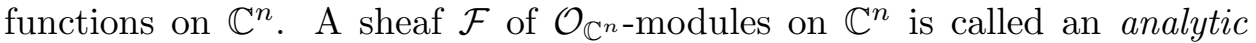
sheaf; if all spaces $\mathcal{F}(U)$ (for $U \subseteq \mathbb{C}^{n}$ open) are Fréchet $\mathcal{O}(U)$-modules, we call $\mathcal{F}$ an analytic Fréchet sheaf.

Definition 2.3. An analytic Fréchet sheaf $\mathcal{F}$ is called quasi-coherent if, for all open Stein subsets $U \subseteq \mathbb{C}^{n}$, the natural restriction and multiplication map

$$
\mathcal{O}(U) \widehat{\otimes}_{\mathcal{O}\left(\mathbb{C}^{n}\right)} \mathcal{F}\left(\mathbb{C}^{n}\right) \rightarrow \mathcal{F}(U)
$$

is an isomorphism of Fréchet spaces, and if

$$
\mathcal{O}(U) \perp_{\mathcal{O}\left(\mathbb{C}^{n}\right)} \mathcal{F}\left(\mathbb{C}^{n}\right) .
$$

Thus, the analytic Fréchet sheaf $\mathcal{F}$ is quasi-coherent if and only if the augmented Bar complex

$$
\mathbf{B}^{\mathcal{O}\left(\mathbb{C}^{n}\right)}\left(\mathcal{O}(U), \mathcal{F}\left(\mathbb{C}^{n}\right)\right) \rightarrow \mathcal{F}(U) \rightarrow 0
$$

is exact for every Stein open subset $U \subseteq \mathbb{C}^{n}$.

Proposition 2.4. Let $E$ be a Fréchet $\mathcal{O}\left(\mathbb{C}^{n}\right)$-module. Then there is a quasi-coherent sheaf such that $E$ can be identified with its space of global sections if and only if

$$
E \perp_{\mathcal{O}\left(\mathbb{C}^{n}\right)} \mathcal{O}(U)
$$

holds for every Stein open subset $U \subseteq \mathbb{C}^{n}$. In this case, the sheaf $\widetilde{E}$, defined by

$$
\widetilde{E}(U):=\mathcal{O}(U) \widehat{\otimes}_{\mathcal{O}\left(\mathbb{C}^{n}\right)} E \quad \text { for } U \subseteq \mathbb{C}^{n} \text { open, }
$$

is the unique quasi-coherent sheaf with the property that $\widetilde{E}\left(\mathbb{C}^{n}\right) \cong E$ as Fréchet $\mathcal{O}\left(\mathbb{C}^{n}\right)$-modules. 
Because of Proposition 2.4, we call a Fréchet $\mathcal{O}\left(\mathbb{C}^{n}\right)$-module $E$ quasicoherent if (2.1) holds for every Stein open subset $U \subseteq \mathbb{C}^{n}$. In this case, $\widetilde{E}$ is called its associated quasi-coherent sheaf.

The next theorem provides a useful criterion for quasi-coherence and yields a number of non-trivial examples.

TheOREM 2.5. Every soft analytic Fréchet sheaf is quasi-coherent.

Corollary 2.6. Let $E$ be a Fréchet $\mathcal{O}\left(\mathbb{C}^{n}\right)$-module. If there exists a soft analytic Fréchet sheaf $\mathcal{F}$ such that $E \cong \mathcal{F}\left(\mathbb{C}^{n}\right)$ as Fréchet $\mathcal{O}\left(\mathbb{C}^{n}\right)$-modules, then $E$ is quasi-coherent and $\widetilde{E}=\mathcal{F}$.

Corollary 2.6 allows us to call a Fréchet $\mathcal{O}\left(\mathbb{C}^{n}\right)$-module $E$ soft if there is a soft analytic Fréchet sheaf $\mathcal{F}$ with $E \cong \mathcal{F}\left(\mathbb{C}^{n}\right)$.

If $E$ is a Fréchet $\mathcal{E}\left(\mathbb{C}^{n}\right)$-module, then the canonical sheaf

$$
U \mapsto \mathcal{O}(U) \widehat{\otimes} E \quad \text { for } U \subseteq \mathbb{C}^{n} \text { open }
$$

is a soft analytic Fréchet sheaf with $E \cong \mathcal{F}\left(\mathbb{C}^{n}\right)$, and hence $E$ is soft.

Let $X$ be a Banach space and $a=\left(a_{1}, \ldots, a_{m}\right)$ a commuting tuple in $L(X)$, the Banach algebra of bounded linear operators on $X$. We equip $X$ with the $\mathcal{O}\left(\mathbb{C}^{n}\right)$-module structure determined by $a$, i.e.

$$
\mathcal{O}\left(\mathbb{C}^{n}\right) \times X \rightarrow X, \quad(f, x) \mapsto f(a) x,
$$

where $f(a) \in L(X)$ is defined in terms of power series. This turns $X$ into a Banach $\mathcal{O}\left(\mathbb{C}^{n}\right)$-module.

Definition 2.7. Let $X$ and $a$ be as above. We say that the tuple $a$ satisfies property $(\beta)$ if the Banach $\mathcal{O}\left(\mathbb{C}^{n}\right)$-module $X$ is quasi-coherent. In this case the associated quasi-coherent sheaf $\widetilde{X}$ is called the canonical sheaf model of $a$.

By Proposition 2.4 the tuple $a$ satisfies property $(\beta)$ if and only if

$$
X \perp_{\mathcal{O}\left(\mathbb{C}^{n}\right)} \mathcal{O}(U)
$$

holds for every Stein open subset $U \subseteq \mathbb{C}^{n}$, or, using the Koszul resolution, if the Koszul complex $\mathbf{K}_{\bullet}(z-a, \mathcal{O}(U, X))$ has separated homology in degree $p=0$ and is exact in degree $p>0$.

If we replace the space $\mathcal{O}(U)$ in the above transversality relation by the Fréchet $\mathcal{O}\left(\mathbb{C}^{n}\right)$-module $\mathcal{E}(U)$, we are led to the following definition.

Definition 2.8. The commuting tuple $a \in L(X)^{m}$ is said to satisfy property $(\beta)_{\mathcal{E}}$ if the Banach $\mathcal{O}\left(\mathbb{C}^{n}\right)$-module $X$ satisfies

$$
X \perp_{\mathcal{O}\left(\mathbb{C}^{n}\right)} \mathcal{E}\left(\mathbb{C}^{n}\right) .
$$

As before, the tuple $a$ satisfies property $(\beta)_{\mathcal{E}}$ if and only if the Koszul complex K. $\left(z-a, X \widehat{\otimes} \mathcal{E}\left(\mathbb{C}^{n}\right)\right)$ has separated homology in degree $p=0$ and 
is exact in degree $p>0$. Using locally finite $C^{\infty}$-partititions of unity, one can show that property $(\beta)_{\mathcal{E}}$ yields

$$
X \perp_{\mathcal{O}\left(\mathbb{C}^{n}\right)} \mathcal{E}(U)
$$

for all $U \subseteq \mathbb{C}^{n}$ open.

A useful criterion for property $(\beta)_{\mathcal{E}}$ is the following.

Theorem 2.9. If $X$ is a Banach $\mathcal{E}\left(\mathbb{C}^{n}\right)$-module, then

$$
X \perp_{\mathcal{O}\left(\mathbb{C}^{n}\right)} \mathcal{E}\left(\mathbb{C}^{n}\right) .
$$

Let $X$ be a Banach $\mathcal{O}\left(\mathbb{C}^{n}\right)$-module and denote by $M_{z}$ the tuple of multiplication operators with the coordinate functions $z_{j}(1 \leq j \leq n)$. Then a simple computation shows that the $\mathcal{O}\left(\mathbb{C}^{n}\right)$-module structure on $X$ determined by $M_{z}$ coincides with the given one. In particular, Theorem 2.9 shows that the tuple $M_{z}$ on a Banach $\mathcal{E}\left(\mathbb{C}^{n}\right)$-module $X$ has property $(\beta)_{\mathcal{E}}$.

2.3. The tangential Cauchy-Riemann complex and Hardy spaces. Let $\Omega \subseteq \mathbb{C}^{n}, n \geq 2$, be a bounded domain with smooth boundary $b \Omega$. For $0 \leq p \leq n$, we consider the tangential Cauchy-Riemann complex

$$
0 \rightarrow \mathfrak{B}^{p, 0}(b \Omega) \stackrel{\bar{\partial}_{b}}{\rightarrow} \mathfrak{B}^{p, 1}(b \Omega) \stackrel{\bar{\partial}_{b}}{\rightarrow} \ldots \stackrel{\bar{\partial}_{b}}{\rightarrow} \mathfrak{B}^{p, n-1}(b \Omega) \rightarrow 0
$$

as defined for instance in [2]. Since the spaces $\mathfrak{B}^{p, q}(b \Omega)$ are defined by a pointwise orthogonality relation, they are invariant under multiplication by $\mathcal{E}\left(\mathbb{C}^{n}\right)$-functions, i.e. $\mathfrak{B}^{p, q}(b \Omega)$ carries the structure of an algebraic $\mathcal{E}\left(\mathbb{C}^{n}\right)$ module. Let $\sigma$ be the surface measure on $b \Omega$ and denote by $L^{2}(b \Omega)$ the corresponding Lebesgue space. We define $L_{q}^{2}(b \Omega)$ to be the completion of $\mathfrak{B}^{0, q}(b \Omega)$ under the sum of the $L^{2}$-norms of the coefficients. Then $L_{q}^{2}(b \Omega)$ is a Hilbert space and we define the operator $T^{q}$ to be the maximal extension of $\bar{\partial}_{b}$, i.e. the domain of $T^{q}$ is

$$
\begin{aligned}
& D^{q}:=\left\{f \in L_{q}^{2}(b \Omega): \exists g \in L_{q+1}^{2}(b \Omega)\right. \text { with } \\
& \left.\qquad \bar{\partial}_{b} f=g \text { in the distribution sense }\right\},
\end{aligned}
$$

and for $f \in D^{q}$ we define

$$
T^{q}(f):=g,
$$

where $g \in L_{q+1}^{2}(b \Omega)$ is any solution of $\bar{\partial}_{b} f=g$. As usual, we use in the following freely the notation $\bar{\partial}_{b}$ for the operator $T^{q}$ as well. It is easy to see that $T^{q}$ is a densely defined closed linear operator between Hilbert spaces.

The kernel of the first operator in the tangential Cauchy-Riemann complex has a special meaning.

Definition 2.10. We define the Hardy space $H^{2}(b \Omega)$ as

$$
H^{2}(b \Omega):=\operatorname{Ker}\left(T^{0}\right) .
$$

Thus, $H^{2}(b \Omega)$ is a closed subspace of $L_{0}^{2}(b \Omega)$ and hence a Hilbert space. 
The fundamental exactness and closed range property of the tangential Cauchy-Riemann operator, which is the basis for the proof of the main result of this article, is formulated in the following theorem, which we reproduce from [15].

TheOREM 2.11. Let $\Omega \subseteq \mathbb{C}^{n}, n \geq 2$, be a bounded pseudoconvex domain with smooth boundary. Then the tangential Cauchy-Riemann complex

$$
0 \rightarrow D^{0} \stackrel{\bar{\partial}_{b}}{\rightarrow} D^{1} \stackrel{\bar{\partial}_{b}}{\rightarrow} \ldots \stackrel{\bar{\partial}_{b}}{\rightarrow} D^{n-1} \rightarrow 0
$$

is exact in degree $0<q<n-1$ and $\operatorname{Im}\left(T^{n-2}\right) \subseteq L_{n-1}^{2}(b \Omega)$ is closed.

We equip the spaces $D^{q}$ with the graph norm $\|\cdot\|_{T^{q}}^{2}:=\|\cdot\|_{L_{q}^{2}(b \Omega)}^{2}+$ $\left\|T^{q}(\cdot)\right\|_{L_{q+1}^{2}(b \Omega)}^{2}$. This turns $D^{q}$ into a Hilbert space for $0 \leq q<n-1$, and because of Theorem 2.11, the quotient

$$
C:=L_{n-1}^{2}(b \Omega) / \operatorname{Im}\left(T^{n-2}\right)
$$

is a Hilbert space as well. This yields the following exact sequence of Hilbert spaces:

$$
0 \rightarrow H^{2}(b \Omega) \hookrightarrow D^{0} \stackrel{\bar{\partial}_{b}}{\rightarrow} D^{1} \stackrel{\bar{\partial}_{b}}{\rightarrow} \ldots \stackrel{\bar{\partial}_{b}}{\rightarrow} D^{n-1} \rightarrow C \rightarrow 0 .
$$

We collect the main properties of the resolution (2.2) in the next proposition. All these statements can easily be verified by using the $\mathcal{E}\left(\mathbb{C}^{n}\right)$-module structure of the spaces $\mathfrak{B}^{0, q}(b \Omega)$ and the properties of the $\bar{\partial}_{b}$-operator.

Proposition 2.12. Let $\Omega \subseteq \mathbb{C}^{n}, n \geq 2$, be a bounded pseudoconvex domain with smooth boundary. Then the resolution (2.2) is an exact sequence in which all spaces are Hilbert and all maps are continuous. Further we have:

- $H^{2}(b \Omega), D^{q}(0 \leq q \leq n-1), Z^{q}:=\operatorname{Im}\left(T^{q-1}: D^{q-1} \rightarrow D^{q}\right)(0<q \leq$ $n-1)$ and $C$ are Hilbert $\mathcal{O}\left(\mathbb{C}^{n}\right)$-modules.

- $D^{q}(0 \leq q \leq n-1)$ is a Hilbert $\mathcal{E}\left(\mathbb{C}^{n}\right)$-module.

We conclude this section with some remarks on the various definitions of Hardy spaces. To simplify our discussion, we assume that the domain $\Omega$ is strictly pseudoconvex with smooth boundary. This allows us to make use of some deep results from function theory in order to prove the equivalence of the above definition of the Hardy space $H^{2}(b \Omega)$ with the others. First we notice that every function in $H^{2}(b \Omega)$ can be approximated by smooth CR functions; a proof of the next lemma can be found in [9].

LEMmA 2.13. Let $\Omega \subseteq \mathbb{C}^{n}, n \geq 2$, be a bounded strictly pseudoconvex domain with smooth boundary. Then

$$
H^{2}(b \Omega)={\overline{\left\{f \in C^{\infty}(b \Omega): \bar{\partial}_{b} f=0\right\}}}^{L^{2}(b \Omega)} .
$$


The following proposition establishes the connection of our Definition 2.10 of the Hardy space $H^{2}(b \Omega)$ with the others.

Proposition 2.14. Let $\Omega \subseteq \mathbb{C}^{n}, n \geq 2$, be a bounded strictly pseudoconvex domain with smooth boundary. Then

$$
H^{2}(b \Omega)={\overline{\left.A(\Omega)\right|_{b \Omega}}}^{L}{ }^{2}(b \Omega),
$$

where $A(\Omega):=\mathcal{O}(\Omega) \cap C(\bar{\Omega})$ is the Banach algebra of continuous functions on $\bar{\Omega}$ that are holomorphic in $\Omega$.

Proof. For $f \in H^{2}(b \Omega)$, Lemma 2.13 gives us a sequence $\left(f_{n}\right)_{n \in \mathbb{N}} \subseteq$ $C^{\infty}(b \Omega)$ with $\bar{\partial}_{b} f_{n}=0$ and $f_{n} \rightarrow f$ in $L^{2}(b \Omega)$. By a generalization of the Hartogs Extension Theorem (Theorem IV.2.5 in [14]) there are functions $F_{n} \in A(\Omega)$ with $\left.F_{n}\right|_{b \Omega}=f_{n}$. This shows $f \in \overline{\left.A(\Omega)\right|_{b \Omega}} L^{2}(b \Omega)$. On the other hand, if $f=\left.F\right|_{b \Omega}$ with $F \in A(\Omega)$, there are functions $F_{n} \in \mathcal{O}(\bar{\Omega})$ with $F_{n} \rightarrow F$ uniformly on $\bar{\Omega}$ (Theorem VII.2.1 in [14]). Then

$$
f_{n}:=\left.F_{n}\right|_{b \Omega} \in C^{\infty}(b \Omega),
$$

$\bar{\partial}_{b} f_{n}=0$ and $f_{n} \rightarrow f$ in $L^{2}(b \Omega)$. This yields $\left.A(\Omega)\right|_{b \Omega} \subseteq H^{2}(b \Omega)$ and since $H^{2}(b \Omega)$ is closed, the missing inclusion is proved.

If $\Omega \subseteq \mathbb{C}^{n}$ is a domain with $C^{2}$ boundary, there are two other equivalent ways to define the Hardy space $\overline{\left.A(\Omega)\right|_{b \Omega}}{ }^{2}(b \Omega)$. In the first one, a function $f \in \mathcal{O}(\Omega)$ belongs to $H^{2}(\Omega)$ if and only if $|f|^{2}$ has a harmonic majorant on $\Omega$. The second and perhaps most familiar definition of the Hardy space is

$$
H^{2}(\Omega):=\left\{f \in \mathcal{O}(\Omega): \sup _{0<\varepsilon<\varepsilon_{0}}\left(\int_{b \Omega_{\varepsilon}}|f|^{2} d \sigma_{\varepsilon}\right)^{1 / 2}<\infty\right\},
$$

where $\Omega_{\varepsilon}$ "approximates" $\Omega$ from inside for $\varepsilon \searrow 0$. For details and complete proofs we refer to the standard literature, for instance [11] and [12].

3. The main result. In this section we state and prove the main result of this article concerning the localization of Hardy spaces. For this purpose we need the following two technical lemmas.

Lemma 3.1. Let $E, F$ be Fréchet spaces and $T: E \rightarrow F$ a continuous linear operator. Then $\operatorname{Im} T \subseteq F$ is closed if and only if for every sequence $\left(e_{n}\right)_{n \in \mathbb{N}} \subseteq E$ with $\lim _{n \rightarrow \infty} T\left(e_{n}\right)=0$ there is a sequence $\left(\widetilde{e}_{n}\right)_{n \in \mathbb{N}} \subseteq \operatorname{Ker} T$ such that $e_{n}-\widetilde{e}_{n} \rightarrow 0$ as $n \rightarrow \infty$.

Pr o of. We consider the induced operator

$$
\widehat{T}: E / \operatorname{Ker} T \rightarrow \operatorname{Im} T
$$

and denote by $q: E \rightarrow E / \operatorname{Ker} T$ the quotient map. If $\operatorname{Im} T$ is closed, the open mapping theorem shows that $S:=(\widehat{T})^{-1}$ is continuous. For a sequence 
$\left(e_{n}\right)_{n \in \mathbb{N}} \subseteq E$ with $\lim T\left(e_{n}\right)=0$ we therefore have $S T\left(e_{n}\right)=q\left(e_{n}\right) \rightarrow 0$. Let $d$ be the translation invariant metric on $E$ and $\widetilde{d}$ the quotient metric $\left({ }^{1}\right)$ on $E / \operatorname{Ker} T$. Then we can find two sequences $\left(\widetilde{e}_{n}\right)_{n \in \mathbb{N}}$ and $\left(\widetilde{\widetilde{e}}_{n}\right)_{n \in \mathbb{N}}$ in $\operatorname{Ker} T$ with

$$
d\left(e_{n}-\widetilde{e}_{n}, \widetilde{\widetilde{e}}_{n}\right) \leq d\left(e_{n}-\widetilde{e}_{n}, \operatorname{Ker} T\right)+1 / n \leq \widetilde{d}\left(q\left(e_{n}\right), 0\right)+2 / n \rightarrow 0 .
$$

Therefore the sequence $\left(\widetilde{e}_{n}+\widetilde{\widetilde{e}}_{n}\right)_{n \in \mathbb{N}} \subseteq \operatorname{Ker} T$ has the desired properties. On the other hand, the given condition yields the continuity of $S$ and this clearly implies the closedness of $\operatorname{Im} T$.

For details on the quotient topology in topological vector spaces we refer to the classical literature, for instance [16].

LEMma 3.2. We consider the following commutative diagram:

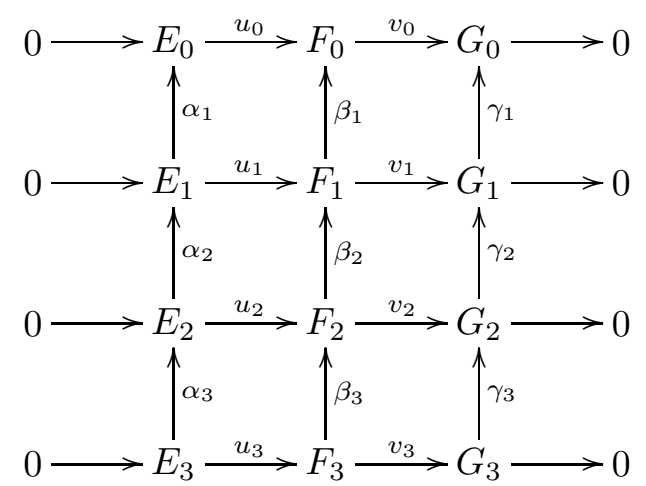

in which all spaces are Fréchet, all maps are continuous linear operators, and we assume the rows are exact, the columns form complexes, $\operatorname{Im} \beta_{1}$ is closed, $\operatorname{Ker} \beta_{1}=\operatorname{Im} \beta_{2}, \operatorname{Im} \gamma_{2}$ is closed and $\operatorname{Ker} \gamma_{2}=\operatorname{Im} \gamma_{3}$. Then $\operatorname{Im} \alpha_{1}$ is closed.

Pro of. Let $\left(e_{1}^{n}\right)_{n \in \mathbb{N}} \subseteq E_{1}$ be a sequence with $\alpha_{1}\left(e_{1}^{n}\right) \rightarrow 0$. According to Lemma 3.1 we have to show that there is a sequence $\left(\widetilde{e}_{1}^{n}\right)_{n \in \mathbb{N}} \subseteq \operatorname{Ker} \alpha_{1}$ with $e_{1}^{n}-\widetilde{e}_{1}^{n} \rightarrow 0$. The convergence $\alpha_{1}\left(e_{1}^{n}\right) \rightarrow 0$ implies $u_{0} \alpha_{1}\left(e_{1}^{n}\right)=\beta_{1} u_{1}\left(e_{1}^{n}\right) \rightarrow 0$ and since $\operatorname{Im} \beta_{1}$ is closed there are $f_{1}^{n} \in \operatorname{Ker} \beta_{1}$ with

$$
u_{1}\left(e_{1}^{n}\right)-f_{1}^{n} \rightarrow 0 \text {. }
$$

$\left({ }^{1}\right)$ For two elements $e_{1}, e_{2} \in E$ we have the following description of the quotient metric on $E / \operatorname{Ker} T$ :

$$
\widetilde{d}\left(q\left(e_{1}\right), q\left(e_{2}\right)\right)=\inf _{\tilde{e} \in \operatorname{Ker} T} d\left(e_{1}-\widetilde{e}, q^{-1}\left(q\left(e_{2}\right)\right)\right),
$$

where

$$
d(e, N):=\inf _{\tilde{\tilde{e}} \in N} d(e, \widetilde{\widetilde{e}})
$$

denotes the distance of the point $e \in E$ to the subset $N \subseteq E$. 
By assumption $\operatorname{Ker} \beta_{1}=\operatorname{Im} \beta_{2}$ and hence we have $f_{1}^{n}=\beta_{2}\left(f_{2}^{n}\right)$ with $f_{2}^{n} \in$ $F_{2}$. This yields

$$
\gamma_{2} v_{2}\left(f_{2}^{n}\right)=v_{1} \beta_{2}\left(f_{2}^{n}\right)=v_{1}\left(f_{1}^{n}\right)-\underbrace{v_{1} u_{1}\left(e_{1}^{n}\right)}_{=0}=v_{1}\left(f_{1}^{n}-u_{1}\left(e_{1}^{n}\right)\right) \rightarrow 0
$$

and the closedness of $\operatorname{Im} \gamma_{2}$ gives the existence of elements $g_{2}^{n} \in \operatorname{Ker} \gamma_{2}$ such that

$$
v_{2}\left(f_{2}^{n}\right)-g_{2}^{n} \rightarrow 0 .
$$

Because of $\operatorname{Ker} \gamma_{2}=\operatorname{Im} \gamma_{3}$ we have $g_{2}^{n}=\gamma_{3}\left(g_{3}^{n}\right)$ with $g_{3}^{n} \in G_{3}$ and the surjectivity of $v_{3}$ yields $g_{3}^{n}=v_{3}\left(f_{3}^{n}\right)$ with $f_{3}^{n} \in F_{3}$. Therefore $\widetilde{f}_{2}^{n}:=\beta_{3}\left(f_{3}^{n}\right)$ has the properties

$$
\beta_{2}\left(\widetilde{f}_{2}^{n}\right)=0 \quad \text { and } \quad v_{2}\left(\widetilde{f}_{2}^{n}\right)=v_{2} \beta_{3}\left(f_{3}^{n}\right)=\gamma_{3} v_{3}\left(f_{3}^{n}\right)=\gamma_{3}\left(g_{3}^{n}\right)=g_{2}^{n} .
$$

Since $v_{2}\left(f_{2}^{n}-\widetilde{f}_{2}^{n}\right)=v_{2}\left(f_{2}^{n}\right)-g_{2}^{n} \rightarrow 0$ and $\operatorname{Im} v_{2}=G_{2}$ is closed there are $u_{2}\left(e_{2}^{n}\right) \in \operatorname{Im} u_{2}=\operatorname{Ker} v_{2}$ with

$$
f_{2}^{n}-\widetilde{f}_{2}^{n}-u_{2}\left(e_{2}^{n}\right) \rightarrow 0
$$

and hence

$$
f_{1}^{n}-u_{1} \alpha_{2}\left(e_{2}^{n}\right)=\beta_{2}\left(f_{2}^{n}\right)-\underbrace{\beta_{2}\left(\widetilde{f}_{2}^{n}\right)}_{=0}-\beta_{2} u_{2}\left(e_{2}^{n}\right) \rightarrow 0 .
$$

This implies

$$
u_{1}\left(e_{1}^{n}-\alpha_{2}\left(e_{2}^{n}\right)\right)=\left(u_{1}\left(e_{1}^{n}\right)-f_{1}^{n}\right)+\left(f_{1}^{n}-u_{1} \alpha_{2}\left(e_{2}^{n}\right)\right) \rightarrow 0
$$

and since $\operatorname{Ker} u_{1}=\{0\}$ and $\operatorname{Im} u_{1}=\operatorname{Ker} v_{1}$ is closed, Lemma 3.1 shows that

$$
e_{1}^{n}-\alpha_{2}\left(e_{2}^{n}\right) \rightarrow 0 \text {. }
$$

Therefore $\widetilde{e}_{1}^{n}:=\alpha_{2}\left(e_{2}^{n}\right)$ has the desired properties and this completes the proof.

We are now able to prove the main result of this article.

Theorem 3.3. Let $\Omega \subseteq \mathbb{C}^{n}, n \geq 2$, be a bounded pseudoconvex domain with smooth boundary. Then the transversality relation

$$
H^{2}(b \Omega) \perp_{\mathcal{O}\left(\mathbb{C}^{n}\right)} \mathcal{E}\left(\mathbb{C}^{n}\right)
$$

holds, i.e. the tuple $M_{z}$ of multiplication operators with the coordinate functions $z_{j}(1 \leq j \leq n)$ has property $(\beta)_{\mathcal{E}}$.

Pr o of. For shortness we use in this proof the abbreviations $\mathcal{E}:=\mathcal{E}\left(\mathbb{C}^{n}\right)$, $H^{2}:=H^{2}(b \Omega)$ and $\mathcal{O}:=\mathcal{O}\left(\mathbb{C}^{n}\right)$. Since $\mathcal{O}$ and $\mathcal{E}$ are nuclear Fréchet spaces, the characteristic property of the Tor-functor (Theorem 2.1), applied to the short exact sequence

$$
0 \rightarrow H^{2} \hookrightarrow D^{0} \stackrel{\bar{\partial}_{b}}{\rightarrow} Z^{1} \rightarrow 0
$$


yields the long exact sequence of locally convex spaces

$$
\begin{aligned}
& 0 \rightarrow \widehat{\operatorname{Tor}}_{n}^{\mathcal{O}}\left(H^{2}, \mathcal{E}\right) \rightarrow \widehat{\operatorname{Tor}}_{n}^{\mathcal{O}}\left(D^{0}, \mathcal{E}\right) \rightarrow \widehat{\operatorname{Tor}}_{n}^{\mathcal{O}}\left(Z^{1}, \mathcal{E}\right) \rightarrow \ldots \\
& \ldots \rightarrow \widehat{\operatorname{Tor}}_{p+1}^{\mathcal{O}}\left(H^{2}, \mathcal{E}\right) \rightarrow \widehat{\operatorname{Tor}}_{p+1}^{\mathcal{O}}\left(D^{0}, \mathcal{E}\right) \rightarrow \widehat{\operatorname{Tor}}_{p+1}^{\mathcal{O}}\left(Z^{1}, \mathcal{E}\right) \rightarrow \ldots \\
& \ldots \rightarrow \widehat{\operatorname{Tor}}_{p}^{\mathcal{O}}\left(H^{2}, \mathcal{E}\right) \rightarrow \widehat{\operatorname{Tor}}_{p}^{\mathcal{O}}\left(D^{0}, \mathcal{E}\right) \rightarrow{\widehat{\operatorname{Tor}_{p}^{\mathcal{O}}}}^{\mathcal{O}}\left(Z^{1}, \mathcal{E}\right) \rightarrow \ldots \\
& \ldots \rightarrow H^{2} \widehat{\otimes}_{\mathcal{O}} \mathcal{E} \quad \rightarrow D^{0} \widehat{\otimes}_{\mathcal{O}} \mathcal{E} \quad \rightarrow Z^{1} \widehat{\otimes}_{\mathcal{O}} \mathcal{E} \quad \rightarrow 0 .
\end{aligned}
$$

By Proposition 2.12, $D^{q}$ is a Hilbert $\mathcal{E}\left(\mathbb{C}^{n}\right)$-module and hence $D^{q} \perp_{\mathcal{O}} \mathcal{E}$ for $0 \leq q \leq n-1$ (Theorem 2.9). In particular, we have $\widehat{\operatorname{Tor}}_{p}^{\mathcal{O}}\left(D^{0}, \mathcal{E}\right)=0$ for all $p \geq 1$ and this shows

$$
\widehat{\operatorname{Tor}}_{p}^{\mathcal{O}}\left(H^{2}, \mathcal{E}\right) \cong \widehat{\operatorname{Tor}}_{p+1}^{\mathcal{O}}\left(Z^{1}, \mathcal{E}\right) \quad \text { as locally convex spaces }
$$

for all $p \geq 1$, and

$$
\widehat{\operatorname{Tor}}_{n}^{\mathcal{O}}\left(H^{2}, \mathcal{E}\right)=0
$$

The same idea, applied to the short exact sequences

$$
0 \rightarrow Z^{q} \hookrightarrow D^{q} \stackrel{\bar{\partial}_{b}}{\rightarrow} Z^{q+1} \rightarrow 0
$$

for $1 \leq q<n-1$ and

$$
0 \rightarrow Z^{n-1} \hookrightarrow D^{n-1} \rightarrow C \rightarrow 0,
$$

yields, for all $p \geq 1$,

$$
\widehat{\operatorname{Tor}}_{p}^{\mathcal{O}}\left(Z^{q}, \mathcal{E}\right) \cong \widehat{\operatorname{Tor}}_{p+1}^{\mathcal{O}}\left(Z^{q+1}, \mathcal{E}\right) \text { and } \widehat{\operatorname{Tor}}_{p}^{\mathcal{O}}\left(Z^{n-1}, \mathcal{E}\right) \cong \widehat{\operatorname{Tor}}_{p+1}^{\mathcal{O}}(C, \mathcal{E}) \text {. }
$$

Together we have

$$
\widehat{\operatorname{Tor}}_{p}^{\mathcal{O}}\left(H^{2}, \mathcal{E}\right) \cong \widehat{\operatorname{Tor}}_{p+1}^{\mathcal{O}}\left(Z^{1}, \mathcal{E}\right) \cong \ldots \cong \widehat{\operatorname{Tor}}_{p+n-1}^{\mathcal{O}}\left(Z^{n-1}, \mathcal{E}\right) \cong \underbrace{\widehat{\operatorname{Tor}}_{p+n}^{\mathcal{O}}(C, \mathcal{E})}_{=0}
$$

for all $p \geq 1$. According to Definition 2.2, it remains to show that $H^{2} \widehat{\otimes}_{\mathcal{O}} \mathcal{E}$ is a Hausdorff locally convex space. We consider the commutative diagram

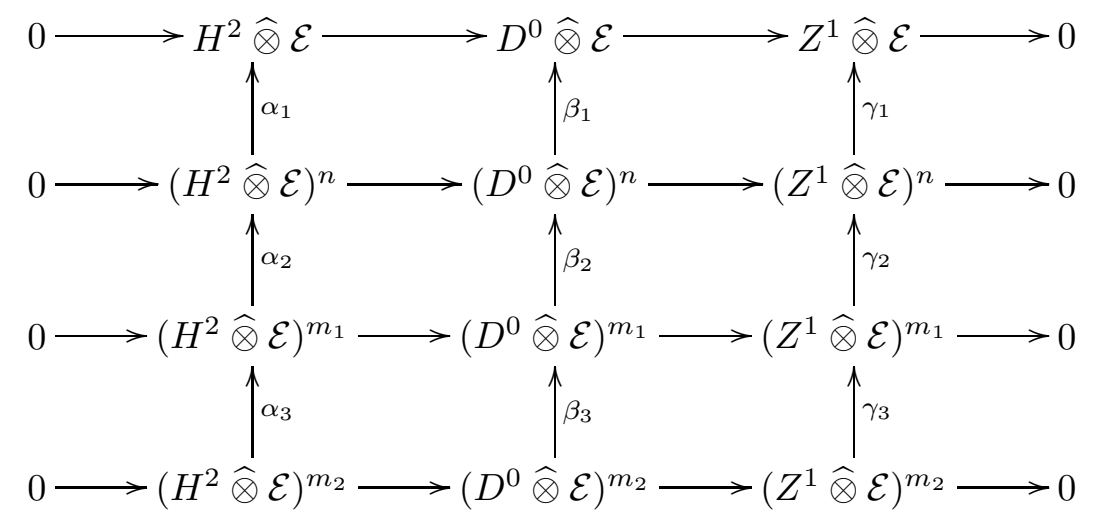

in which the rows arise from tensoring with $\mathcal{E}$ and the columns are given by the respective Koszul resolution $\mathbf{K}_{\bullet}\left(z-w, H^{2} \widehat{\otimes} \mathcal{E}\right), m_{j}=\left(\begin{array}{c}m \\ j\end{array}\right)$ for $j=1,2$. 
In this diagram, all spaces are Fréchet, all maps are continuous linear operators, the rows are exact and the columns form complexes. Because the isomorphisms

$$
\widehat{\operatorname{Tor}}_{1}^{\mathcal{O}}\left(Z^{1}, \mathcal{E}\right) \cong \widehat{\operatorname{Tor}}_{2}^{\mathcal{O}}\left(Z^{2}, \mathcal{E}\right) \cong \ldots \cong \widehat{\operatorname{Tor}}_{n}^{\mathcal{O}}(C, \mathcal{E})
$$

are topological and $\widehat{\operatorname{Tor}}_{n}^{\mathcal{O}}(C, \mathcal{E})$ is separated, we see that $\widehat{\operatorname{Tor}}_{1}^{\mathcal{O}}\left(Z^{1}, \mathcal{E}\right)=$ $\operatorname{Ker}\left(\gamma_{1}\right) / \operatorname{Im}\left(\gamma_{2}\right)$ is separated and hence

$$
\operatorname{Im}\left(\gamma_{2}\right) \text { is closed. }
$$

By $D^{0} \perp_{\mathcal{O}} \mathcal{E}$ we find that

$$
\operatorname{Im}\left(\beta_{1}\right) \text { is closed and } \operatorname{Ker}\left(\beta_{1}\right)=\operatorname{Im}\left(\beta_{2}\right),
$$

and, finally, $\widehat{\operatorname{Tor}}_{2}^{\mathcal{O}}\left(Z^{1}, \mathcal{E}\right)=0$ implies

$$
\operatorname{Ker}\left(\gamma_{2}\right)=\operatorname{Im}\left(\gamma_{3}\right) \text {. }
$$

Now, Lemma 3.2 shows that $\operatorname{Im}\left(\alpha_{1}\right)$ is closed and this is equivalent to the fact that $H^{2} \widehat{\otimes}_{\mathcal{O}} \mathcal{E}=\left(H^{2} \widehat{\otimes} \mathcal{E}\right) / \operatorname{Im}\left(\alpha_{1}\right)$ is a Hausdorff locally convex space. This proves Theorem 3.3.

Since property $(\beta)_{\mathcal{E}}$ implies property $(\beta)$, we have the following corollary.

Corollary 3.4. Let $\Omega \subseteq \mathbb{C}^{n}, n \geq 2$, be a bounded pseudoconvex domain with smooth boundary. Then the Hilbert $\mathcal{O}\left(\mathbb{C}^{n}\right)$-module $H^{2}(b \Omega)$ is quasicoherent.

Let $\Omega \subseteq \mathbb{C}^{n}, n \geq 2$, be a bounded strictly pseudoconvex domain with smooth boundary $b \Omega$, and let $\mathcal{F}$ denote the associated quasi-coherent sheaf of $H^{2}(b \Omega)$. Then we have the following description of $\mathcal{F}$ :

Let $r$ be a smooth strictly plurisubharmonic defining function for $\Omega$, defined on an open neighborhood $U$ of $\bar{\Omega}$, i.e.

$$
\Omega=\{z \in U: r(z)<0\} \quad \text { and } \quad b \Omega=\{z \in U: r(z)=0\} .
$$

Then, for a sufficiently small $\varepsilon_{0}>0$ and $0<\varepsilon<\varepsilon_{0}$, the set

$$
\Omega_{\varepsilon}=\{z \in U: r(z)<-\varepsilon\}
$$

is again a bounded strictly pseudoconvex domain with smooth boundary

$$
b \Omega_{\varepsilon}=\{z \in U: r(z)=-\varepsilon\},
$$

and it approximates $\Omega$ from inside.

Because the maximal ideal space of the Banach algebra $A(\Omega)=\mathcal{O}(\Omega) \cap$ $C(\bar{\Omega})$ coincides with $\bar{\Omega}$, we have the identity $\sigma\left(M_{z}, H^{2}(b \Omega)\right)=\bar{\Omega}$ for the Taylor spectrum of the multiplication tuple $M_{z}$ on $H^{2}(b \Omega)$ (see for instance [18]). $\mathcal{F}$ is the sheaf model of $M_{z}$ and has therefore the support $\sigma\left(M_{z}, H^{2}(b \Omega)\right)$. This shows that $\left.\mathcal{F}\right|_{\mathbb{C}^{n} \backslash \bar{\Omega}}=0$. The global section space $\mathcal{F}\left(\mathbb{C}^{n}\right)=H^{2}(b \Omega)$ consists of analytic functions in $\Omega$ with certain growth conditions at the boundary. Therefore it is clear that $\left.\mathcal{F}\right|_{\Omega}=\left.\mathcal{O}_{\mathbb{C}^{n}}\right|_{\Omega}$; this 
identity can be proved formally by using known techniques of Fredholm theory for several commuting operators (compare with [7], Section 4.3). It remains to describe the stalk $\mathcal{F}_{P}$ of the sheaf $\mathcal{F}$ at a point $P \in b \Omega$.

According to the general theory of Fréchet soft analytic sheaves, presented in [7], Section 4.4, it is clear how to localize the Hilbert $\mathcal{E}\left(\mathbb{C}^{n}\right)$ modules $D^{0}, D^{1}, \ldots, D^{n-1}$. More exactly, if $\mathcal{D}^{j}$ denotes the quasi-coherent sheaf which localizes $D^{j}$, then $u \in \mathcal{D}_{P}^{j}$ if and only if there is an open neighborhood $U$ in $b \Omega$ of the point $P$ and a test function $\varphi \in \mathcal{D}(U)$ with the property that $\varphi \cdot u \in D^{j}$ and $\varphi=1$ in a neighborhood of $P$. Since the localization functor is exact, we obtain

$$
\mathcal{F}_{P}=\operatorname{Ker}\left(\bar{\partial}_{b}: \mathcal{D}_{P}^{0} \rightarrow \mathcal{D}_{P}^{1}\right) .
$$

In order to describe this kernel we observe that an element $u \in \mathcal{D}_{P}^{0}$ satisfies $\bar{\partial}_{b} u=0$ and can therefore be extended locally to an analytic function $f$ inside $\Omega$. For this, let $U$ be an open neighborhood of $P$ in $\mathbb{C}^{n}$ such that $u$ is defined on $U \cap b \Omega$ and it extends analytically to $U \cap \Omega$ (for details see [9], Theorem 4.3). Let $\Omega^{\prime}$ be a strictly pseudoconvex domain with smooth boundary such that $\Omega^{\prime} \subset \Omega \cap U$ and $b \Omega^{\prime} \cap b \Omega$ contains an open neighborhood of $P$ in $b \Omega$. Let $r^{\prime}$ be a smooth strictly plurisubharmonic defining function for $\Omega^{\prime}$; we can assume that $r=r^{\prime}$ in a neighborhood $B$ of $P$ in $\mathbb{C}^{n}$. According to (2.3) we have

$$
\sup _{\varepsilon \searrow 0} \int_{r^{\prime}(z)=-\varepsilon}|f(z)|^{2} d \sigma_{\varepsilon}^{\prime}(z)<\infty .
$$

In particular, this yields

$$
\sup _{\varepsilon \backslash 0} \int_{\substack{z \in B \\ r(z)=-\varepsilon}}|f(z)|^{2} d \sigma_{\varepsilon}(z)<\infty .
$$

Conversely, if an analytic function $f \in \mathcal{O}(U \cap \Omega)$ satisfies condition (3.1) for an open neighborhood $B$ of the point $P \in b \Omega$, then the preceding argument can be reversed with minor modifications, and we obtain as boundary values of $f$ an element $u \in \mathcal{D}_{P}^{0}$ such that $\bar{\partial}_{b} u=0$ and $u$ extends analytically to $f$ in a neighborhood of $P \in b \Omega$.

In conclusion we obtain the following description of the sheaf $\mathcal{F}$ at a boundary point $P \in b \Omega$ :

$\mathcal{F}_{P}=\left\{f \in \mathcal{O}\left(B_{f} \cap \Omega\right): B_{f}\right.$ open neighborhood of $P$ in $\mathbb{C}^{n}$,

$$
\left.\sup _{\varepsilon \searrow 0} \int_{\substack{z \in B_{f} \\ r(z)=-\varepsilon}}|f(z)|^{2} d \sigma_{\varepsilon}(z)<\infty\right\} .
$$

This result is in accord with the similar description of the sheaf which localizes the Bergman space of a pseudoconvex domain; cf. [7], Chapter 8. 
4. Applications. In this last section of the present paper, we collect some applications of the theory developed in the first three sections. All of them are derived analogously to the Bergman case, treated in Chapter 8 of [7]. We only sketch the ideas and proofs, a complete and precise treatment will be given in the second author's Ph.D. thesis.

4.1. Tuples $T_{f}$ with $f^{\prime} \in H^{\infty}$ have property $(\beta)$. As a first application we show that the tuple of multiplication operators on $H^{2}(b \Omega)$ with symbols in a certain class of functions has property $(\beta)$.

If $\Omega \subseteq \mathbb{C}^{n}$ is a bounded open set, we denote the Banach algebra of bounded holomorphic functions on $\Omega$ by $H^{\infty}(\Omega)$. The space

$$
\begin{aligned}
H_{1}^{\infty}(\Omega):=\left\{f \in H^{\infty}(\Omega):\right. & \\
& \text { all partial derivatives of } f \text { are bounded on } \Omega\}
\end{aligned}
$$

is clearly a closed subalgebra of $H^{\infty}(\Omega)$ and hence a Banach algebra itself. If $\Omega$ is in addition strictly pseudoconvex with smooth boundary $b \Omega$, then every $f \in H^{\infty}(\Omega)$ has non-tangential boundary limit at almost every point of $b \Omega$, and the limit function $\widetilde{f}$ belongs to $L^{\infty}(b \Omega)$. Then the Hardy space $H^{2}(b \Omega)$ is a Hilbert $H^{\infty}(\Omega)$-module. Further, the spaces $D^{q}(0 \leq q \leq n-1)$ and $C$ in the resolution (2.2) are Hilbert $H_{1}^{\infty}(\Omega)$-modules, as one can prove directly from the definitions.

We denote the commuting tuple of multiplication operators on $H^{2}(b \Omega)$ with $f=\left(f_{1}, \ldots, f_{m}\right) \in\left(H^{\infty}(\Omega)\right)^{m}$ by $T_{f}=\left(T_{f_{1}}, \ldots, T_{f_{m}}\right)$. If we restrict the symbol $f$ to the subalgebra $H_{1}^{\infty}(\Omega)$, we have the following result.

TheOREM 4.1. Let $\Omega \subseteq \mathbb{C}^{n}, n \geq 2$, be a bounded strictly pseudoconvex domain with smooth boundary. If $m \leq n$ and $f \in\left(H_{1}^{\infty}(\Omega)\right)^{m}$, the commuting tuple $T_{f}$ on $H^{2}(b \Omega)$ has property $(\beta)$.

Pro of. We consider the pull-back map

$$
f^{*}: \mathcal{O}\left(\mathbb{C}^{m}\right) \rightarrow H_{1}^{\infty}(\Omega)
$$

that maps $F \in \mathcal{O}\left(\mathbb{C}^{m}\right)$ onto the function $f^{*}(F):=F \circ f$ in $H_{1}^{\infty}(\Omega)$. Since $f(\Omega) \subseteq \mathbb{C}^{m}$ is bounded, $f^{*}$ is well defined and clearly a continuous homomorphism of algebras. Therefore, every Banach $H_{1}^{\infty}(\Omega)$-module $X$ becomes a Banach $\mathcal{O}\left(\mathbb{C}^{m}\right)$-module with the module structure determined by $f^{*}$, i.e.

$$
\mathcal{O}\left(\mathbb{C}^{m}\right) \times X \rightarrow X, \quad(F, x) \mapsto f^{*}(F) x .
$$

It is easy to check that the module structure determined by $f^{*}$ on $X$ coincides with the module structure on $X$ determined by the tuple $T_{f} \in(L(X))^{m}$ of multiplication operators (cf. Section 2.2). Clearly, every $H_{1}^{\infty}(\Omega)$-module homomorphism becomes an $\mathcal{O}\left(\mathbb{C}^{n}\right)$-module homomorphism. Thus, the tuple $T_{f}$ has property $(\beta)$ if and only if $X$, equipped with the $\mathcal{O}\left(\mathbb{C}^{m}\right)$-module structure determined by $f^{*}$, is quasi-coherent. 
Because (2.2) is an exact sequence in the category of Hilbert $H_{1}^{\infty}(\Omega)$ modules, we obtain the exact sequence

$$
0 \rightarrow H^{2}(b \Omega) \hookrightarrow D^{0} \stackrel{\bar{\partial}_{b}}{\rightarrow} D^{1} \stackrel{\bar{\partial}_{b}}{\rightarrow} \ldots \stackrel{\bar{\partial}_{b}}{\rightarrow} D^{n-1} \rightarrow C \rightarrow 0
$$

of Hilbert $\mathcal{O}\left(\mathbb{C}^{m}\right)$-modules. In this resolution, the spaces $D^{q}(0 \leq q \leq n-1)$ are soft, because $f$ induces in a similar way an $\mathcal{E}\left(\mathbb{C}^{m}\right)$-module structure on each $D^{q}$. Therefore, if $m=n$, the $\mathcal{O}\left(\mathbb{C}^{m}\right)$-module $H^{2}(b \Omega)$ is quasi-coherent by Corollary 4.4.5 in [7]. If $m<n$, we cut the resolution (4.1) to the right length, i.e. we consider the exact sequence

$$
0 \rightarrow H^{2}(b \Omega) \hookrightarrow D^{0} \stackrel{\bar{\partial}_{b}}{\rightarrow} D^{1} \stackrel{\bar{\partial}_{b}}{\rightarrow} \ldots \stackrel{\bar{\partial}_{b}}{\rightarrow} D^{m-1} \rightarrow D^{m} / \operatorname{Im}\left(\bar{\partial}_{b}\right) \rightarrow 0,
$$

and apply the same corollary to this resolution. This proves Theorem 4.1.

4.2. Quasi-similarity of tuples $T_{f}$ as in 4.1. We recall that two bounded linear operators $T$ and $S$ acting on the Banach spaces $X$ and $Y$ are called quasi-similar if there are two injective bounded linear operators with dense range $A: X \rightarrow Y$ and $B: Y \rightarrow X$ such that $A T=S A$ and $B S=T B$. In the last two decades, it was proved by different methods that this equivalence relation preserves the various spectra of certain classes of operators.

A unifying approach to these phenomena, which at the same time allows for the generalization to commuting tuples of operators, is based on the observation that a large part of the concrete classes of operators can be characterized by Bishop's property $(\beta)$.

We cite the following theorem from [13].

Theorem 4.2. Let $T=\left(T_{1}, \ldots, T_{m}\right)$ and $S=\left(S_{1}, \ldots, S_{m}\right)$ denote two commuting $m$-tuples of bounded linear operators acting on the Banach spaces $X, Y$, respectively. Assume that there exist bounded linear operators with dense range $A: X \rightarrow Y$ and $B: Y \rightarrow X$ such that $A T_{j}=S_{j} A$ and $B S_{j}=T_{j} B$ for $j=1, \ldots, m$. If both tuples $T$ and $S$ have Bishop's property $(\beta)$, then their Taylor and essential Taylor spectra coincide:

$$
\sigma(T, X)=\sigma(S, Y) \quad \text { and } \quad \sigma_{\mathrm{ess}}(T, X)=\sigma_{\mathrm{ess}}(S, Y) .
$$

Since the operators $A$ and $B$ in Theorem 4.2 are not required to be injective, this theorem holds under a slightly weaker condition than the quasi-similarity. However, for most applications the quasi-similarity relation is important.

As in the Bergman case, we immediately obtain the next corollary.

Corollary 4.3. Let $\Omega \subseteq \mathbb{C}^{n}$ and $\Omega^{\prime} \subseteq \mathbb{C}^{n^{\prime}}, n, n^{\prime} \geq 2$, be two bounded strictly pseudoconvex domains with smooth boundaries, and let $f=\left(f_{1}, \ldots\right.$ $\left.\ldots, f_{m}\right)$ and $g=\left(g_{1}, \ldots, g_{m}\right), m \leq \min \left(n, n^{\prime}\right)$, be two tuples in $H_{1}^{\infty}(\Omega)$, $H_{1}^{\infty}\left(\Omega^{\prime}\right)$, respectively. If the tuples $T_{f}=\left(T_{f_{1}}, \ldots, T_{f_{m}}\right)$ and $T_{g}=$ 
$\left(T_{g_{1}}, \ldots, T_{g_{m}}\right)$, acting on the Hardy spaces $H^{2}(b \Omega), H^{2}\left(b \Omega^{\prime}\right)$, respectively, are quasi-similar, then

$$
\sigma\left(T_{f}, H^{2}(b \Omega)\right)=\sigma\left(T_{g}, H^{2}\left(b \Omega^{\prime}\right)\right)
$$

and

$$
\sigma_{\mathrm{ess}}\left(T_{f}, H^{2}(b \Omega)\right)=\sigma_{\mathrm{ess}}\left(T_{g}, H^{2}\left(b \Omega^{\prime}\right)\right) .
$$

4.3. Finite-codimensional invariant subspaces. Let $\Omega \subseteq \mathbb{C}^{n}, n \geq 2$, be a bounded strictly pseudoconvex domain with smooth boundary. A subspace $S \subseteq H^{2}(b \Omega)$ is called analytically invariant if it is invariant under all Toeplitz operators with symbols in $H^{\infty}(\Omega)$, i.e.

$$
f \cdot S:=\{f u: u \in S\} \subseteq S \text { for all } f \in H^{\infty}(\Omega) .
$$

There are two natural examples of analytically invariant subspaces of $H^{2}(b \Omega)$. First, we consider spaces of the form

$$
I \cdot H^{2}(b \Omega):=\operatorname{cl} \operatorname{span}\left\{\varphi u: \varphi \in I, u \in H^{2}(b \Omega)\right\}
$$

for an ideal $I \subseteq \mathcal{O}(\bar{\Omega})$. Here cl span $S$ denotes the closed linear span of the set $S$.

The second class of analytically invariant subspaces consists of spaces of the form

$$
S(V):=\left\{u \in H^{2}(b \Omega):\left.u\right|_{V}=0\right\},
$$

where $V$ is a closed subset of $\Omega$. Clearly, $I \cdot H^{2}(b \Omega)$ and $S(V)$ are invariant under multiplication with $f \in H^{\infty}(\Omega)$.

We show in this section that every analytically invariant subspace of finite codimension in $H^{2}(b \Omega)$ belongs to the first class; more precisely, we have the following theorem.

Theorem 4.4. Let $\Omega \subseteq \mathbb{C}^{n}, n \geq 2$, be a bounded strictly pseudoconvex domain with smooth boundary. If $S \subseteq H^{2}(b \Omega)$ is an analytically invariant subspace of finite codimension, then there are polynomials $P_{1}, \ldots, P_{m}$ (in $\left.z_{1}, \ldots, z_{n}\right)$ with only finitely many zeroes inside $\Omega$ such that

$$
S=\sum_{j=1}^{m} P_{j} \cdot H^{2}(b \Omega) .
$$

Thus, $S=I \cdot H^{2}(b \Omega)$, where $I$ is the ideal in $\mathcal{O}(\bar{\Omega})$ generated by $P_{1}, \ldots, P_{m}$.

Proof. We consider the quotient space $Q:=H^{2}(b \Omega) / S$. Then $Q$ is a Fréchet $\mathcal{O}\left(\mathbb{C}^{n}\right)$-module of finite dimension, and has therefore a finite support that is contained in $\bar{\Omega}$. Moreover, it can be shown that the support is contained in $\Omega$.

By Corollary 3.4, the Hilbert $\mathcal{O}\left(\mathbb{C}^{n}\right)$-module $H^{2}(b \Omega)$ is quasi-coherent, and the same is true for the Fréchet $\mathcal{O}\left(\mathbb{C}^{n}\right)$-module $Q$. Let $\mathcal{F}$ and $\mathcal{Q}$ denote their associated quasi-coherent sheaves. A detailed description of the sheaf 
$\mathcal{F}$ was given at the end of Section 3. $\operatorname{Since} \operatorname{supp}(\mathcal{Q})$ is finite, Hilbert's Syzyzy Theorem yields a global finite resolution

$$
0 \rightarrow \mathcal{O}_{\mathbb{C}^{n}}^{m_{r}} \rightarrow \ldots \rightarrow \mathcal{O}_{\mathbb{C}^{n}}^{m_{1}} \rightarrow \mathcal{O}_{\mathbb{C}^{n}} \rightarrow \mathcal{Q} \rightarrow 0
$$

in which the boundaries are induced by polynomials $P_{1}, \ldots, P_{m}$. Because $\operatorname{supp}(\mathcal{Q}) \subseteq \Omega$ and $\left.\mathcal{F}\right|_{\Omega}=\left.\mathcal{O}_{\mathbb{C}^{n}}\right|_{\Omega}$, we can obtain a new exact sequence by tensoring this complex with $\widehat{\otimes}_{\mathcal{O}_{\mathbb{C}}} \mathcal{F}$ :

$$
0 \rightarrow \mathcal{F}^{m_{r}} \rightarrow \ldots \rightarrow \mathcal{F}^{m_{1}} \rightarrow \mathcal{F} \rightarrow \mathcal{Q} \rightarrow 0 .
$$

Both sheaves $\mathcal{F}$ and $\mathcal{Q}$ are acyclic on $\mathbb{C}^{n}$, and hence the corresponding sequence of global sections

$$
0 \rightarrow H^{2}(b \Omega)^{m_{r}} \rightarrow \ldots \rightarrow H^{2}(b \Omega)^{m_{1}} \rightarrow H^{2}(b \Omega) \rightarrow Q \rightarrow 0
$$

remains exact. This shows that $S$ is the image of the last differential in this complex, which is of the desired type. This proves Theorem 4.4.

Theorem 4.4 connects the finite-codimensional analytically invariant subspaces of $H^{2}(b \Omega)$ with a certain class of polynomial ideals $I \subset \mathbb{C}\left[z_{1}, \ldots, z_{n}\right]$. This can be used to classify these invariant subspaces modulo isomorphisms of topological $\mathcal{O}\left(\mathbb{C}^{n}\right)$-modules.

If $V \subseteq \Omega$ is closed and we denote by $I(V)$ the closed ideal of functions in $\mathcal{O}(\bar{\Omega})$ that vanish on $V$,

$$
I(V):=\left\{f \in \mathcal{O}(\bar{\Omega}):\left.f\right|_{V}=0\right\},
$$

then $I(V) \cdot H^{2}(b \Omega) \subseteq S(V)$. Another question that could be treated in this context is under what conditions these two spaces coincide.

4.4. Rigidity of Hardy submodules. The classification of all analytically invariant subspaces of the Hardy space of a domain in several complex variables is far from being as simple and as well understood as the classification given by Beurling's theorem in the case of the unit disc. An important series of results in this unknown territory was recently obtained by Ronald G. Douglas and his school; see [4] and [5]. By exploiting our main result (the quasi-coherence of the Hardy space) and some classical algebraic facts, we obtain below a rigidity theorem which complements similar results from [4] and [5]. In the sequel we closely follow the proof of Theorem 8.4.2 in [7] (which is the same result for the Bergman space). Consequently, we skip some technical details and a (more than necessary) preparation.

Let $\Omega \subseteq \mathbb{C}^{n}$ be a bounded strictly pseudoconvex domain with smooth boundary. Let $\mathcal{J} \subseteq \mathcal{O}_{\bar{\Omega}}$ be a coherent sheaf of ideals which satisfies the following two conditions:

(i) $\mathcal{O}(\bar{\Omega}) / \mathcal{J}(\bar{\Omega}) \perp_{\mathcal{O}(\bar{\Omega})} H^{2}(b \Omega)$,

(ii) $\mathcal{J}(\bar{\Omega}) \cdot H^{2}(b \Omega)=\left\{f \in H^{2}(b \Omega):\left.f\right|_{\Omega} \in \mathcal{J}(\Omega)\right\}$. 
The sheaf $\mathcal{O} / \mathcal{J}$ is called in that case privileged with respect to the Hardy space $H^{2}(b \Omega)$ - a notion introduced and studied in analytic geometry by A. Douady; see [3].

If the ideal $\mathcal{J} \subseteq \mathcal{O}_{\bar{\Omega}}$ satisfies conditions (i) and (ii), then we infer via known results of analytic geometry (the structure of coherent sheaves and Hilbert's Syzygy Theorem) that there is a finite exact complex (of $\mathcal{O}(\bar{\Omega})$ modules) of the form

$$
0 \rightarrow H^{2}(b \Omega) \rightarrow \ldots \rightarrow H^{2}(b \Omega)^{p_{0}} \rightarrow H^{2}(b \Omega) \stackrel{\text { res }}{\longrightarrow} \mathcal{O}(\Omega) / \mathcal{J}(\Omega) \rightarrow 0 .
$$

See for details [7], §8.4.

Theorem 4.5. Let $\Omega \subseteq \mathbb{C}^{n}, n \geq 2$, be a bounded strictly pseudoconvex domain with smooth boundary and let $\mathcal{J}_{1}, \mathcal{J}_{2} \subseteq \mathcal{O}_{\bar{\Omega}}$ be two coherent ideals satisfying conditions (i) and (ii). Suppose that $\max _{j=1,2}\left(\operatorname{dim}_{\mathbb{C}} \operatorname{supp} \mathcal{O}_{\bar{\Omega}} / \mathcal{J}_{j}\right)$ $\leq n-2$. Then the (closed) analytic subspaces $\mathcal{J}_{1}(\bar{\Omega}) \cdot H^{2}(b \Omega)$ and $\mathcal{J}_{2}(\bar{\Omega})$. $H^{2}(b \Omega)$ are isomorphic as topological $\mathcal{O}\left(\mathbb{C}^{n}\right)$-modules if and only if $\mathcal{J}_{1}=\mathcal{J}_{2}$.

Proof. The condition in the statement and the preceding resolution show that the analytic modules $\mathcal{J}_{j}(\bar{\Omega}) \cdot H^{2}(b \Omega)(j=1,2)$ are quasi-coherent. Let $\mathcal{F}_{j}(j=1,2)$ be the corresponding Fréchet analytic sheaves. Since the localization of $H^{2}(b \Omega)$ coincides with the sheaf of analytic functions for points $\lambda \in \Omega$, we obtain $\mathcal{F}_{j, \lambda}=\mathcal{J}_{j, \lambda}(j=1,2 ; \lambda \in \Omega)$. Therefore $\mathcal{J}_{1, \lambda} \cong \mathcal{J}_{2, \lambda}$ (as $\mathcal{O}_{\lambda}$-modules) for all $\lambda \in \Omega$. The support condition then implies (via a non-trivial algebraic result of Grothendieck) that $\mathcal{J}_{1, \lambda}=\mathcal{J}_{2, \lambda}$ for all $\lambda \in \Omega$ (see for details [7], Lemma 8.3.3, or [5]).

In conclusion, $\mathcal{J}_{1}(\Omega)=\mathcal{J}_{2}(\Omega)$; but condition (ii) implies that $\mathcal{J}_{1}(\bar{\Omega})$. $H^{2}(b \Omega)=\mathcal{J}_{2}(\bar{\Omega}) \cdot H^{2}(b \Omega)$ and the proof is complete.

\section{References}

[1] E. Bishop, A duality theorem for an arbitrary operator, Pacific J. Math. 9 (1959), 379-394.

[2] A. Boggess, CR Manifolds and the Tangential Cauchy-Riemann Complex, CRC Press, Boca Raton, Fla., 1991.

[3] A. Douady, Le problème des modules pour les sous-espaces analytiques compacts d'un espace analytique donné, Ann. Inst. Fourier (Grenoble) 16 (1) (1966), 1-95.

[4] R. G. Douglas and V. Paulsen, Hilbert Modules over Function Algebras, Pitman Res. Notes Math. Ser. 219, Harlow, 1989.

[5] R. G. Douglas, V. Paulsen, C. H. Sah, and K. Yan, Algebraic reduction and rigidity for Hilbert modules, Amer. J. Math. 117 (1995), 75-92.

[6] N. Dunford and J. T. Schwartz, Linear Operators, Part III, Wiley-Interscience, New York, 1971.

[7] J. Eschmeier and M. Putinar, Spectral Decompositions and Analytic Sheaves, London Math. Soc. Monographs, Oxford Univ. Press, Oxford, 1996. 
[8] C. Foiaş, Spectral maximal spaces and decomposable operators in Banach space, Arch. Math. (Basel) 14 (1963), 341-349.

[9] G. M. Henkin, H. Lewy's equation and analysis on pseudoconvex manifolds, Russian Math. Surveys 32 (1977), 59-130 (transl. from Uspekhi Mat. Nauk 32 (3) (1977), 57-118).

[10] J. J. Kohn, The range of the tangential Cauchy-Riemann operator, Duke Math. J. 53 (1986), 525-545.

[11] S. G. Krantz, Function Theory of Several Complex Variables, Wadsworth, Belmont, Calif., 1992.

[12] - Partial Differential Equations and Complex Analysis, CRC Press, Boca Raton, Fla., 1992.

[13] M. Putinar, Quasi-similarity of tuples with Bishop's property $(\beta)$, Integral Equations Operator Theory 15 (1992), 1047-1052.

[14] R. M. Range, Holomorphic Functions and Integral Representations in Several Complex Variables, Grad. Texts in Math. 108, Springer, New York, 1986.

[15] M.-C. Shaw, Local solvability and estimates for $\bar{\partial}_{b}$ on CR manifolds, in: Proc. Sympos. Pure Math. 52, Amer. Math. Soc., Providence, R.I., 1991, 335-345.

[16] F. Treves, Topological Vector Spaces, Distributions and Kernels, Academic Press, New York, 1967.

[17] F. H. Vasilescu, Analytic Functional Calculus and Spectral Decompositions, Reidel, Dordrecht, 1982.

[18] R. Wolff, Spectra of analytic Toeplitz tuples on Hardy spaces, Bull. London Math. Soc., to appear.

Department of Mathematics

University of California

Riverside, California 92521

U.S.A.

E-mail: mputinar@math.ucr.edu
Mathematisches Institut Westfälische Wilhelms-Universität 48149 Münster

Germany E-mail: wolffro@math.uni-muenster.de

Current address:

Fachbereich Mathematik Universität des Saarlandes 6600 Saarbrücken Germany 